\title{
Violencia de género: actitud y conocimiento del personal de salud de Nicaragua
}

\author{
Rosibel de los Angeles Rodríguez-Bolaños, M en ASS, ${ }^{(1)}$ Margarita Márquez-Serrano, M en MS, ${ }^{(1)}$ \\ María de la Luz Kageyama-Escobar, MSP.(1)
}

\section{Rodríguez-Bolaños RA, Márquez-Serrano M, Kageyama-Escobar ML. Violencia de género: actitud y conocimiento del personal de salud de Nicaragua. Salud Publica Mex 2005;47:134-144.}

\section{Resumen}

Objetivo. Determinar la actitud del personal de salud en la identificación y la referencia de las victimas de violencia de género (VG), así como los factores relacionados con dicha actitud. A simismo, conocer las barreras para tal identificación y referencia, y evaluar el nivel de conocimiento sobre las $\mathrm{N}$ ormas y Procedimientos para laA tención de laViolencia Intrafamiliar entre el personal de salud del Ministerio de Salud de N icaragua (M IN SA). Material y métodos Se realizó un estudio transversal entre el personal de salud de 5 de los 17 Sistemas Locales de Atención Integral en Salud (SILAIS) de N icaragua: médicos, enfermeras y auxiliares de enfermería $(n=213)$ del Programa de Atención Integral a la Mujer, $N$ iñez y A dolescencia en el primer nivel de atención del MIN SA, durante los meses de abril a junio de 2003. La actitud se midió de acuerdo con una escala tipo Likert y se construyó un índice de conocimiento sobre las normas de atención. La información se obtuvo por medio de un instrumento de autoaplicación, basado en el cuestionario de un estudio que se realizó entre el personal del Instituto Mexicano de Seguro Social (IMSS) de Morelos, México. Se utilizó un modelo de regresión lo gística para evaluar la asociación de la actitud con diversos factores, así como con el conocimiento sobre las normas de atención. Resultados La actitud de rechazo hacia la VG fue de $76.06 \%$. En el análisis multivariado, los facto res asociados con la actitud de rechazo fueron la profesión médica (RM 6.5, IC 95\% 2.70-15.82), al igual que los niveles medio (RM 4.3, IC 95\% 1.87-10.26) y

\author{
Rodríguez-Bolaños RA, Márquez-Serrano M, \\ Kageyama-Escobar ML. \\ Gender based violence: Knowledge and attitudes \\ of health care providers in Nicaragua. \\ Salud Publica Mex 2005;47:134-144.
}

\begin{abstract}
A bstract
Objective.To assess the knowledge and attitudes of health care personnel towards the identification and referral of gender-based violence victims (GBV). Also, to identify barriers to identification and referral of GBV, and to assess the levels of knowledge about Norms and Procedures for Intra-Family Violence Care by the health care personnel of the N icaraguan's Minister of Health (MIN SA, for its initials in Spanish). Material and Methods A cross-sectional study was conducted among healthcare professionals, including physicians, nurses and nursing technical aides $(n=213)$, in 5 of the 17 Local Systems of Integral Attention (SILAIS) from the Integral Program of Attention for Women, Children and Ado lescence (AIMNA) in the primary level of attention in MIN SA, from A pril to June 2003. Attitude was measured with a Likert scale and an awareness index was created for intra-family violence care guidelines. The information was obtained using a self-administered instrument, based on the questionnaire of the study made among the personnel of the Mexican Institute for So cial Security (IMSS, for its initials in Spanish), Morelos, Mexico.A logistic regression model was used to evaluate the association between attitude and several factors, as well as with the knowledge of care guidelines. Results In our population, $76.06 \%$ showed an attitude opposing GBV. In the multivariate analysis, the factors associated with opposition to GBV were: medical profession (O R $6.5,95 \% \mathrm{Cl}$ 2.70-15.82), having a middle (O R $4.3,95 \% \mathrm{Cl} 1.87-10.26)$ or high level (OR $3.3,95 \% \mathrm{Cl}$
\end{abstract}

(1) Centro de Investigación en Sistemas de Salud, Instituto N acional de Salud Pública (IN SP). Cuernavaca, Morelos, México.

Fecha de recibido: 28 de noviembre de 2003 - Fecha de aceptado: 10 de febrero de 2005 
alto (RM 3.3, IC 95\% 1.03-10.75) de conocimiento sobre las normas de atención y la cercanía de familiares 0 amigos que han sido víctimas de violencia (RM 3.2, IC 95\% 1.56-6.80). La escasa capacitación sobre el tema (59.9\%), el temor a involucrarse en asuntos legales (52.6\%) y el carácter privado de la violencia $(50.7 \%)$ constituyen las barreras más importantes para la identificación y la referencia de las víctimas. Conclusiones En general, el personal de salud presentó valores altos en la actitud de rechazo hacia laVG. Sin embargo, se identificaron barreras que indican la persistencia de creencias tradicionales como la de considerar el problema de la violencia un asunto privado. Por esta razón, para que en la práctica se observe un cambio significativo, es importante que se consolide la capacitación sobre el tema con una perspectiva de género en las escuelas de medicina. Los hallazgos que se obtuvieron en el presente estudio permitirán mejorar el modelo de atención en los servicios de salud del primer nivel de atención de $\mathrm{N}$ icaragua.

Palabras claves: violencia de género; personal de salud; violencia doméstica; actitud; cono cimiento; N icaragua
1.03-10.75) of knowledge about intra-family violence guidelines and the closeness to relatives or friends who were victims of gender violence $(O \mathrm{R} 3.2,95 \% \mathrm{Cl}$ 1.56-6.80). The lack of training on the subject (59.9\%), fear of getting involved in legal issues (52.6\%), and the concept that violence is a private affair and not a social one $(50.7 \%)$, constituted the most important barriers to providing medical care. Conclusions The health care personnel generally were observed to have high values in regard to an attitude of rejection towards GBV. However, we found barriers that show persisting traditional beliefs, such as considering violence to be a personal issue.Therefore, in order, to ensure a substantial improvement, better training about this subject is needed in medical school curricula with an emphasis on the gender perspective. The finding of the present study will allow improvements in health care reforms at the first level of care in the health sector in N icaragua.

Key words: gender based violence; health care personnel; domestic violence; attitude; awareness; $N$ icaragua
$\mathrm{L}$ a violencia de género (VG) es considerada como un problema de salud pública ${ }^{1}$ que conlleva a daños a la salud física y mental de las mujeres, y por tanto es un factor de demanda de los servicios de salud, lo que representa un costo real para la sociedad.

Dentro de las implicaciones que este problema tiene para la salud de las mujeres se encuentran: las lesiones físicas directas, la inflamación pélvica aguda, un mayor riesgo de contagio de enfermedades de transmisión sexual y VIH/SIDA, embarazos no deseados, aborto, parto prematuro, bajo peso al nacer, entre otras. ${ }^{2-6}$ Dentro de las implicaciones de la violencia en la salud mental se encuentran: depresión, ansiedad, baja autoestima, disfunción sexual, etcétera. ${ }^{7,8}$

Según un informe del Banco Mundial, de 1993, las mujeres ganan, por la VG, 9.5 años de vida con discapacidad (AVAD) ${ }^{9}$ y pierden $20 \%$ de años de vida saludable (AVISA). ${ }^{10}$ Los costos que se generan dentro de los servicios de salud en el ámbito mundial por este problema se calcularon en aproximadamente $5 \%$ del producto interno bruto (PIB). ${ }^{11}$ En el caso de Nicaragua los costos son de 29.5 millones de dólares, equivalentes a 1.6\% del PIB de $1996 .{ }^{12} \mathrm{La}$ violencia de género es un problema frecuente. Estudios en Nicaragua ${ }^{8}$ han documentado que 1 de cada 2 mujeres había sido golpeada por su compañero alguna vez en la vida, y 1 de cada 4 había sido víctima de violencia física en los últimos 12 meses. Morrison ${ }^{12}$ refirió, en 1997, que 70\% de las mujeres había sufrido violencia física alguna vez en su vida, y 33\%, en el año previo al estudio; asimismo, 53\% de las mujeres de la capital (Managua) había sufrido agresiones psicológicas, físicas o sexuales, y $28 \%$, violencia física severa. Los datos de la Encuesta Nicaragüense de Demografía y Salud ${ }^{13}$ indican que $29 \%$ de las mujeres en unión libre había sido víctima de algún abuso físico y sexual en sus vidas.

Es frecuente que en los servicios de salud no se logre identificar el problema de violencia subyacente, aun cuando ésta sea la raíz de los problemas físicos o psicosomáticos por los que las víctimas demandan la atención. Un estudio realizado en Estados Unidos de América (EUA) ${ }_{1}^{14}$ estima que $50 \%$ del personal médico y $70 \%$ del de enfermería consideran que la prevalencia de la VG es equivalente o menor a $1 \%$, y que 1 de cada 10 sujetos del personal médico y 5 de cada 10 del personal de enfermería nunca han identificado una víctima. De acuerdo con datos del Instituto Mexicano de Investigación de Familia y Población en la Ciudad de México, ${ }^{3}$ entre 77 y $88 \%$ del personal de salud desconoce la existencia de protocolos de atención a víctimas de violencia; sin embargo, identifican entre 1 y 5 casos por mes.

Las barreras para la identificación y la referencia de las víctimas de violencia son múltiples y, dependiendo de su origen, se pueden eliminar por medio de intervenciones para la capacitación del personal o la 
reorganización de los servicios. Méndez y colaboradores ${ }^{15}$ realizaron un estudio entre el personal médico del primero y segundo nivel de atención, e identificaron como barreras la falta de privacidad, el corto tiempo de la consulta y la ausencia de protocolos de atención. Waalen y colaboradores ${ }^{16}$ coinciden en que hace falta capacitar al personal, dotarlo de información sobre su responsabilidad y desarrollar intervenciones efectivas; aun cuando existen protocolos o guías de atención se requiere de capacitación sistemática y permanente, como lo afirman Gerbert y colaboradores, ${ }^{17}$ quienes ahondan en las dificultades asociadas con este problema.

Otras barreras difíciles de eliminar están relacionadas con ciertas condiciones sociales, culturales y personales que, desde la perspectiva de género, son cruciales para comprender el problema. Hablar de este tema, por ejemplo, significa introducirse en el espacio privado, por lo que ni las víctimas ni el personal de salud lo hacen. ${ }^{16}$ Un estudio cualitativo realizado por Hathaway y colaboradores ${ }^{18}$ entre mujeres víctimas de VG, atendidas en el Hospital General de Massachussets, concluye que la persona que vive la violencia presenta falta de autoestima, lo que le impide tomar decisiones y tener autodeterminación sobre su vida; siente temor ante el agresor y desesperanza, lo que le dificulta enfrentar su problema.

Las tendencias sexistas, ciertas creencias de orden sociocultural y profesional, así como algunas costumbres y actitudes, impiden al personal de salud visualizar el problema de la VG. Estas actitudes marcan una diferencia significativa en el manejo integral de las víctimas, de acuerdo con Riquer, ${ }^{19}$ quien indagó sobre las percepciones del personal de salud respecto a los obstáculos de atención; esta investigadora coincide con Novack y colaboradores, ${ }^{20}$ quienes se refieren a la promoción de actividades de autoayuda para fomentar la reflexión y la conciencia entre el personal de salud en el abordaje de la VG. Es importante resaltar que el personal de salud también puede ser víctima de VG, lo que dificulta la detección y la referencia de pacientes. ${ }^{21}$

En Nicaragua, para dar respuesta al problema de la VG, desde los años ochenta comenzaron a gestarse organizaciones que promovían la igualdad de derechos para las mujeres, y ello dio lugar a la formación de organizaciones no gubernamentales (ONG). También fue necesario brindar protección a la integridad física y moral de las víctimas, por lo que se crearon las Comisarías de la Mujer (1993), las cuales forman parte de la estructura de la Policía Nacional. Es así como se van integrando otros sectores del Estado y se conforma un modelo de atención de forma intersectorial e interdisciplinaria, del cual el sector salud forma parte.
En el Ministerio de Salud (MINSA) se implementó un modelo de atención a la violencia intrafamiliar, ${ }^{*}$ del cual, desde 1995, se hizo una prueba piloto en el departamento de Estelí. Actualmente, la responsabilidad de la ejecución y del seguimiento del modelo en el primer nivel es la Dirección de Atención Integral a la Mujer y la Niñez en cada uno de los Sistemas Locales de Atención Integral en Salud (SILAIS), y el personal del Programa de Atención Integral a la Mujer, Niñez y Adolescencia (AIMNA) es el encargado de dar la atención a las víctimas que se presentan en los servicios de salud. Para abordar el problema y poder generar acciones que contribuyan a resolverlo se elaboraron las Normas y Procedimientos para la Atención de Violencia Intrafamiliar ${ }^{\ddagger}(2001)$ y el instructivo para el Abordaje Integral de la Violencia Doméstica desde los Servicios de Salud (2002), ${ }^{9,22}$

El objetivo del presente estudio fue determinar la actitud del personal de salud en la identificación y la referencia de las víctimas de VG, al igual que los factores relacionados con esa actitud. Asimismo, conocer las barreras para tal identificación y referencia, y evaluar el nivel de conocimiento sobre las normas y procedimientos para la atención de la violencia intrafamiliar entre en el personal de salud del MINSA.

\section{Material y métodos}

Se realizó un estudio transversal. La población de estudio quedó conformada por todo el personal de salud: el médico, el de enfermería y auxiliares de enfermería $(\mathrm{N}=219)$ que laboraban en 5 de los 17 SILAIS (Managua, Masaya, Estelí, Rivas y Chontales) del Programa AIMNA, en el primer nivel de atención del MINSA. Los criterios de inclusión para los SILAIS fueron: una concentración mayor a 50\% de la población (2 259 443), número de centros de salud en los departamentos (los que corresponden a 61) y del personal de salud del programa, número elevado de usuarias de entre 10 a 40 años de edad, ${ }^{23}$ y una alta prevalencia de violencia. Estelí se eligió por ser el sitio donde se implantó la prueba piloto y en Chontales porque aún no se ha aplicado el modelo de atención. El criterio de inclusión del personal de salud fue pertenecer al Programa de AIMNA. El estudio se llevó a cabo de abril a junio de 2003, con el personal que se encontraba en su puesto. La recolección de la información se hizo con el consentimiento informado y

\footnotetext{
* Decreto Ministerial N 67-6, noviembre de 1996.

‡ Se utilizarán las siglas VIF por ser el nombre oficial de las Normas.
} 
verbal del personal de salud; la participación de este último fue voluntaria y anónima. El cuestionario se diseñó para ser autoaplicado, y se entregó y recibió en sobre cerrado, con base en los principios de Helsinki.

El cuestionario se basó en el estudio realizado entre el personal médico del Instituto Mexicano de Seguro Social (IMSS) en Morelos, México, sobre el conocimiento y la actitud hacia la violencia contra la mujer. ${ }^{15} \mathrm{Al}$ cuestionario original se le realizaron modificaciones para adaptarlo al contexto nicaragüense. Se eliminaron algunas preguntas y se agregaron otras sobre las Normas y los Protocolos para la Atención de las Víctimas de VIF de Nicaragua. Se aplicó el cuestionario para su validación al personal que labora en una ONG con 13 años de experiencia en atención a víctimas de VG.

La variable dependiente fue la actitud con sus tres componentes: ${ }^{15} \mathrm{el}$ cognitivo (lo que uno piensa), el afectivo (lo que uno siente) y el conductual (tendencia a manifestar los pensamientos y las emociones). Para este trabajo se tomó la definición de Allport, ${ }^{15}$ quien se refiere a la actitud como un: "Estado mental y neural de disposición organizado a través de la experiencia, que ejerce una influencia directiva o dinámica sobre la conducta del individuo ante todos los objetos y situaciones con los que se relaciona". Sin embargo, hay que mencionar el aspecto de colectividad que tiene la actitud, el cual es expresión del entorno social en el que se desenvuelve el individuo. Como lo menciona Casales, ${ }^{24}$ la actitud constituye una forma de orientación del proceder de las personas, dependiente de la norma grupal, y tiene la propiedad de reflejarse en el comportamiento del individuo, de orientarlo, regularlo y guiarlo en determinado sentido; de esta manera, es posible distinguir en toda actitud un objetivo, una dirección y una intensidad.

La actitud se midió de acuerdo con una escala tipo Likert de seis puntos, la cual se construyó a partir de 36 reactivos. Se estableció un puntaje mínimo de 36 y un máximo de 216. La escala se operacionalizó de forma dicotómica con un puntaje de corte de 150, que corresponde a la media:

Actitud desfavorable (0): con puntuación $\leq 150$, e incluye al personal que aprueba la VG.

Actitud favorable (1): con puntuación > 150, e incluye al personal que rechaza la VG.

Se complementaron los componentes cognitivo y conductual con 11 preguntas relacionadas con el proceso de atención a las mujeres víctimas de violencia.

Las variables independientes fueron:
1. Datos sociodemográficos: edad, sexo, estado civil, profesión, años de práctica médica, años de antigüedad en el MINSA, tiempo en el Programa de AIMNA (10 preguntas).

2. Información sobre capacitación acerca de VG (cuatro preguntas).

3. Importancia y temas de capacitación sobre VG (dos preguntas).

4. Evaluación del conocimiento sobre las Normas y Procedimientos para la Atención de la Violencia Intrafamiliar, para lo cual se construyó un índice de conocimiento con 13 preguntas; la puntuación mínima era de 0 , y la máxima, de 18, dividida homogéneamente en tres categorías:

Nivel de conocimiento bajo (0): sujetos con puntuación de 0 a 9.09 .

Nivel de conocimiento medio (1): sujetos con puntuación de 9.10 a 13.09 .

Nivel de conocimiento alto (2): sujetos con puntuación de 13.10 a 18.

5. Información sobre experiencia de $\mathrm{VG}$ en personas cercanas o familiares, o bien sobre la propia (cuatro preguntas).

Se indagó acerca de las opiniones que el personal de salud tiene sobre cuáles son las barreras institucionales y de la práctica médica que existen en los servicios de salud para identificar y referir los casos de mujeres víctimas de violencia (cuatro preguntas).

Se realizó análisis bivariado mediante tablas de contingencia y prueba de ji ${ }^{2}$. Se aplicó el análisis de regresión logística múltiple, obteniendo estimadores de razón de momios (RM) con intervalo de confianza de 95\% (IC 95\%). Se incluyeron las variables que en el análisis bivariado tuvieron un valor de $p \leq 0.20$ : profesión, ubicación del centro de salud, conoce las normas, capacitación sobre éstas y el nivel de conocimiento sobre las normas, identificación, referencia y número de casos de víctimas de VG y experiencia de VG en familiares o amigos. También se incluyeron otras variables como edad y sexo. Se evaluaron interacciones, eliminándose variables por no resultar significativas y confusoras. Finalmente, se aplicó la prueba de bondad de ajuste de Pearson, para evaluar la adecuación del modelo. El programa estadístico utilizado en el análisis fue STATA 7.

\section{Resultados}

Participaron 213 sujetos, con una tasa de respuesta de 97.2\%. Las principales características del personal de 
salud en estudio fueron las siguientes: $55.9 \%$ correspondió al personal de enfermería (incluidas las auxiliares), y $44.1 \%$ al personal médico. La participación del SILAIS Managua fue la mayor, con 36.2\%, y 81.6\% de todos los SILAIS pertenecían al área urbana. La edad promedio fue de $34 \pm 7.14$ años, con un rango de 18 a 62 años. El $77.5 \%$ correspondía al sexo femenino y $60.2 \%$ de todos los participantes estaban casados o en unión libre. Respecto a los años de antigüedad en el MINSA, $71 \%$ había trabajado por más de 10 años en el lugar y $39.1 \%$ se encontraba en el Programa de AIMNA desde su inicio (1998); no obstante, 30.1\% del personal llevaba menos de dos años laborando en él. El personal que trabaja en el Programa de Atención a la Mujer equivalía a 36.6\%. El 49.3\% del personal era responsable de alguna área del Programa (cuadro I).

Uno de los hallazgos más importantes fue que la actitud de rechazo hacia la VG estaba presente en $76.06 \%$ del personal de salud. Respecto al componente cognitivo de la actitud resultó que la percepción del personal de salud sobre la magnitud del problema de la VG es que, en promedio, $65.3 \%( \pm 18.4 \%$ con un intervalo de 10 a $98 \%$ ) de las mujeres sufren violencia; $58.1 \%$, daños psicológicos, y un menor porcentaje, daños físicos y sexuales.

En relación con el comportamiento que el personal de salud tiene en la práctica para identificar los casos de violencia se encontró que $44.5 \%$ hace preguntas a las pacientes sólo cuando tiene sospechas; $81.5 \%$ refiere haber identificado casos de VG independientemente de haberlos notificado al sistema de información; de éstos, 38.5\% había identificado de uno a tres casos en los tres meses previos al estudio. El 59.9\% refirió algún caso: a la Comisaría de la Mujer (48.8\%) y al psicólogo (46.8\%). El porcentaje del personal que resultó más apropiado para identificar los casos fue similar entre el personal médico y el de enfermería (41.9\% vs. $40.9 \%$ ) (cuadro II).

Por otra parte, los resultados arrojaron que $60.9 \%$ del personal de salud tenía amigos o familiares que sufrían VG; $32.2 \%$ refirió haber sido víctima de VG durante su infancia, y entre los principales tipos de violencia mencionaron la psicológica (28.7\%), la física (27.2\%) y la sexual (9.3\%); en $62.9 \%$ de los casos, los padres fueron los principales ejecutores. El 30.3\% respondió que en ese momento era víctima de violencia por parte de su compañero/a. El 53\% se refirió a la violencia psicológica; $25 \%$, a la física/psicológica; $9.5 \%$, a la física, y 9.5\%, a los tres tipos. La proporción del personal de salud que en el momento del estudio ejercía VG hacia su pareja fue de $20 \%$, y la violencia psicológica

\section{Cuadro I \\ Características del personal de salud DE LOS SISTEMAS LOCALES DE ATENCIÓN A LA SALUd en estudio. Nicaragua, 2003}

\begin{tabular}{|c|c|}
\hline Característica & Porcentaje \\
\hline Sistemas Locales de Atención Integral en Salud & \\
\hline - Managua & 36.2 \\
\hline - Masaya & 18.3 \\
\hline - Estelí & 10.3 \\
\hline - Rivas & 21.6 \\
\hline - Chontales & 13.6 \\
\hline
\end{tabular}

Edad (años)

- Media y desviación estándar (intervalo) $34 \pm 7.14 /(18$ a 62)

Mujer

Estado civil

- Casado o en unión libre $\quad 60.2$

- Soltero 30.9

- Divorciado, separado o viudo 8.9

Personal

- Médico 44.1

- Enfermería 31.5

- Auxiliar de enfermería 24.4

- Con especialidad (Maestría) 4.7

Años de antigüedad en el MIN SA (más de 10 años) 71

Personal en el Programa de AIMNA

- Desde el inicio (1998) 39.1

- Desde hace dos años

Programa en el que labora

- Mujer 36.6

\begin{tabular}{ll}
\hline Niñez & 29.6 \\
\hline
\end{tabular}

Personal responsable del Programa

49.3

Fuente: C uestionarios del estudio acerca de actitud y conocimiento sobre violencia de género del personal de salud. Ministerio de Salud de N icaragua, 2003

fue la más frecuente (47.8\%), seguida de la física/ psicológica $(29.1 \%)$, la física $(20.9 \%)$ y la sexual $(2.2 \%)$.

Al evaluar el nivel de conocimiento sobre las Normas para la Atención de VIF se encontró que 19.8\% tenía poco conocimiento; $59.6 \%$, medio, y $20.6 \%$, alto, y el personal médico fue el que presentó el nivel más alto $(63.6 \%)$. El porcentaje del personal de salud que conocía las Normas de Atención a Víctimas fue de $47.9 \%$, pero solamente $37.5 \%$ las había leído. El $35.7 \%$ había recibido capacitación sobre las Normas y $85 \%$ la 


\section{Cuadro II \\ Acciones que realiza el PeRsonal de SALUd de los SISTEMAS LOCALES DE ATENCIÓN A LA SALUd EN ESTUDIO DURANTE LA ATENCIÓN A MUJERES VÍCTIMAS DE Violencia de gÉnero. Nicaragua, 2003}

\begin{tabular}{|c|c|c|}
\hline Acciones & $\mathrm{N}^{\circ}$ & Porcentaje \\
\hline Realiza preguntas para identificar un caso & & \\
\hline - Sólo cuando sospecha que se trata de un caso & 94 & 44.5 \\
\hline - Siempre & 50 & 23.7 \\
\hline - Raramente & 51 & 24.1 \\
\hline - Nunca & 16 & 7.6 \\
\hline Ha identificado casos de mujeres víctimas deVG & & \\
\hline en los últimos tres meses & 172 & 81.5 \\
\hline - Ninguno & 76 & 35.7 \\
\hline - De uno a tres & 82 & 38.5 \\
\hline - De cuatro a más & 55 & 25.8 \\
\hline El papel del personal en la identificación de los casos & & \\
\hline - No estar interesado en identificar los casos & 1 & 0.4 \\
\hline - Estar interesado, pero sin hacer algo que ayude & & \\
\hline a las mujeres violentadas & 5 & 2.3 \\
\hline - Estar interesado y hacer lo que el caso amerite & 197 & 92.4 \\
\hline - Otro & 10 & 4.69 \\
\hline Ha referido algún caso de víctima de VG & 127 & 59.9 \\
\hline - Lugar de referencia & & \\
\hline - Psicólogo (a) & 59 & 46.8 \\
\hline - Trabajadora social & 31 & 24.6 \\
\hline - Programa dentro del MINSA & 27 & 21.4 \\
\hline - 0 tra institución u ONG especializada & & \\
\hline en estos casos & 49 & 38.8 \\
\hline - Comisaría de la Mujer & 61 & 48.8 \\
\hline
\end{tabular}

Después de identificar los casos el manejo que el personal ha dado es*

\begin{tabular}{lrr}
- & 152 & 72.0 \\
\hline - Las refiere a profesionales & 14 & 6.6 \\
\hline No les pregunta & 1 & 0.4 \\
\hline - Nonversar con las pacientes tratando de ayudarlas & 189 & 89.5 \\
\hline No sabe qué hacer en este tipo de casos & 3 & 1.4
\end{tabular}

Personal más apropiado para identificar los casos

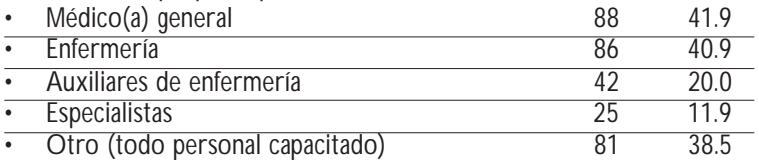

Respondió correctamente a tres tipos de daño que ha identificado en víctimas de VG

\begin{tabular}{lrr}
- Daño físico & 102 & 55.7 \\
\hline - Daño psicológico & 104 & 58.1 \\
\hline Daño sexual & 62 & 38.5
\end{tabular}

* En caso de no haber identificado algún caso, se preguntó cómo lo trataría y referiría

VG: violencia de género; MIN SA: Ministerio de Salud; 0 N G: organización no gubernamental

Fuente: $C$ uestionarios del estudio acerca de actitud y conocimiento sobre violencia de género del personal de salud. Ministerio de Salud de N icaragua, 2003 consideró adecuada. De los que se capacitaron, $60 \%$ había recibido una capacitación; $26.4 \%$, dos, y $13.6 \%$, más de tres.

Asimismo, 96\% del personal refirió que es muy importante la capacitación sobre VG, y entre los temas que les interesaban están: las Normas de Atención y Procedimientos para la Atención de VIF (38\%); historia, definición, ciclo, tipos y consecuencias de la VG, entre otros $(18 \%)$; leyes y los derechos humanos de las mujeres $(15 \%)$; temas de autoestima y defensa ante una situación propia de violencia (13\%); enfoque de género (7\%); violencia contra la niñez y los ancianos (5\%); otros temas relacionados (4\%).

Entre los factores relacionados con la actitud de rechazo están: laborar en los centros de salud del área urbana $(p=0.053)$, haber recibido capacitación sobre las normas de atención $(p=0.082)$, ser personal médico $(p<0.00)$, contar con un nivel medio-alto de conocimiento $(p=0.000)$ y conocer a personas cercanas víctimas de violencia $(p=0.000)$ (cuadro III).

En el análisis de regresión logística se encontró que es 6.5 veces mayor la posibilidad de que el personal médico muestre una actitud de rechazo hacia la VG en comparación con el de enfermería. Hay 4.3 veces mayor posibilidad de que el personal con nivel medio de conocimiento sobre las Normas de Atención tenga una actitud de rechazo, comparado con el personal con conocimiento bajo. Igualmente, es 3.2 veces mayor la posibilidad de que el personal de salud que conoce a gente cercana con experiencia de VG, tenga una actitud de rechazo, en contraste con el personal que no tiene personas cercanas en esa situación (cuadro IV).

\section{Barreras ante la identificación y la referencia de VG}

Entre las barreras institucionales para identificar los casos de VG estuvieron la poca capacitación que existe sobre el tema (59.9\%) y la falta de privacidad en la consulta $(50.4 \%)$, mientras que para referir, además de la poca capacitación $(53.5 \%)$, se mencionó el temor a involucrarse en asuntos legales (52.6\%). Los principales factores que dificultaron la identificación de casos en la práctica médica fueron: el carácter privado de la violencia $(50.7 \%)$ y el temor a que la propia seguridad se vea amenazada (37.8\%); por su parte, lo que dificultó la referencia fue, de nuevo, el resguardo de la propia seguridad $(37.5 \%)$ y el desconocimiento de lugares de referencia (35.6\%). También fue posible observar que a los miembros del personal médico (varones) "no les gusta identificar los casos (...) hay pocos especialistas para atender a las víctimas y también la propia experiencia de VG en el personal" (cuadro V). 


\section{Cuadro III \\ Análisis bivariado de LOS Factores Relacionados CON LA ACTITUD DE RECHAZO HACIA LA VIOLENCIA DE GÉNERO* POR PARTE DEL PERSONAL DE SALUD de los Sistemas Locales de Atención Integral en Salud. Nicaragua, 2003}



Capacitación sobre las N ormas de Atención de las Víctimas de VIF

\begin{tabular}{lll} 
- No capacitado & $99(72.2)$ & \\
\hline - Sí capacitado & $63(82.8)$ & 0.082
\end{tabular}

$\mathrm{N}$ ivel de conocimiento sobre las $\mathrm{N}$ ormas de Atención de las Víctimas de VIF

\begin{tabular}{|c|c|c|}
\hline - $\quad \mathrm{N}$ ivel bajo & $21(50.0)$ & \\
\hline - $\quad$ Nivel medio & $103(81.1)$ & 0.000 \\
\hline - $\quad N$ ivel alto & $38(86.3)$ & 0.000 \\
\hline
\end{tabular}

Identificación de casos de mujeres víctimas de VG

\begin{tabular}{llll} 
- No ha identificado & $21(53.8)$ & \\
\hline - Sí ha identificado & $140(81.4)$ & 0.000
\end{tabular}

Referencia de casos de mujeres víctimas deVG

\begin{tabular}{lrl} 
- No ha referido & $53(62.3)$ & \\
\hline - Sí ha referido & $109(85.8)$ & 0.000
\end{tabular}

N úmero de casos identificados en los últimos tres meses

\begin{tabular}{lrl} 
- 0 casos identificados & $47(61.8)$ & \\
\hline - 1 a más casos identificados & $115(83.9)$ & 0.000
\end{tabular}

Experiencia de VG en familiares 0 amigos

\begin{tabular}{lrl} 
- No presenta & $51(62.2)$ & \\
\hline Sí presenta & $109(85.1)$ & 0.000
\end{tabular}

* Variables que mostraron significancia estadística mediante la prueba de $\chi^{2}$

SILAIS: Sistemas Locales de Atención Integral en Salud VG : violencia de género

Fuente: $C$ uestionarios del estudio acerca de actitud y conocimiento sobre violencia de género del personal de salud. Ministerio de Salud de N icaragua, 2003

\section{Cuadro IV \\ Modelo multivariado de Regresión logística ENTRE ACTITUd DE RECHAZO (FAVORABLE) HACIA LA VG Y Variables independientes.* Personal de salud de los Sistemas Locales de Atención Integral en Salud. Nicaragua, 2003}

Variables RM RM * (Cruda) (Ajustada) Valor de p

Profesión

- Lic. en enfermería y auxiliares de enfermería

- Lic. en medicina

\begin{tabular}{lll}
$1^{\ddagger}$ & $1^{\ddagger}$ & - \\
\hline 6.0 & 6.5 & 0.000
\end{tabular}

$\mathrm{N}$ ivel de conocimiento

\begin{tabular}{lllll} 
- & Bajo & $1^{\ddagger}$ & $1^{\ddagger}$ & \\
\hline - & Medio & 4.2 & 4.3 & 0.001 \\
\hline - Alto & 6.3 & 3.3 & 0.043
\end{tabular}

Experiencia de VG en personas cercanas

\begin{tabular}{llll} 
- N o presentan & $1^{\ddagger}$ & $1^{\ddagger}$ & - \\
\hline - Sí presentan & 3.4 & 3.2 & 0.002
\end{tabular}

* Ajustado por las variables contenidas en el cuadro

¥ Categoría de referencia

Ajuste: prueba de bondad de ajuste Pearson. $\chi^{2}=11.74, p=0.1095$

VG: violencia de género

RM: razón de momios

Fuente: $C$ uestionarios del estudio acerca de actitud y conocimiento sobre violencia de género del personal de salud. Ministerio de Salud de N icaragua, 2003

\section{Discusión}

Es innegable que el personal de salud se encuentra en una posición privilegiada para identificar situaciones de violencia en las mujeres que acuden a los servicios de salud; sin embargo, no siempre se hace y quizás éste sea el único lugar donde las víctimas se presenten. Son varios los factores implicados en esa situación, por lo que es necesario analizarlos con detalle. Uno de los más importantes es precisamente la actitud de rechazo o de aceptación que el personal tenga ante la violencia de género, pero no es el único.

En la presente investigación, los resultados indican que la gran mayoría del personal de salud $(76.06 \%)$ que labora en el programa tiene una actitud de rechazo hacia la VG, lo cual es un buen indicador per se, si se considera que el impacto que puede tener una actitud de aceptación es el de perpetuar y legitimar la VG; ${ }^{14}$ 


\section{Cuadro V \\ BarReras institucionales Y de La práctica médica PARA IDENTIFICAR Y REFERIR LOS CASOS DE VIOLENCIA de género. Personal de salud de los Sistemas Locales de Atención Integral en Salud. Nicaragua, 2003}

Barreras

N Porcentaje

Institucionales para identificar casos de VG

\begin{tabular}{llrr} 
- & Poca capacitación sobre el tema & 127 & 59.9 \\
\hline - & Falta de privacidad en la consulta & 107 & 50.4 \\
\hline - & Falta de tiempo en la consulta & 89 & 41.9 \\
\hline - Temor a involucrarse en asuntos legales & 83 & 39.1 \\
\hline
\end{tabular}

Institucionales para referir

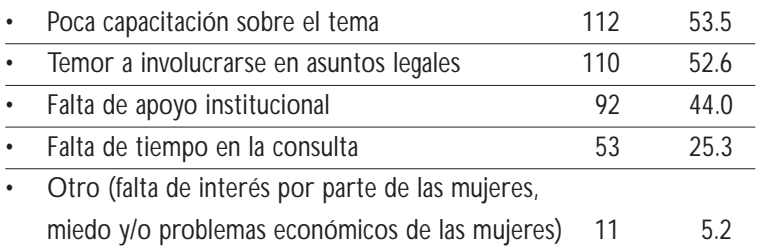

En la práctica médica para identificar

\begin{tabular}{llrl} 
- El carácter privado de la violencia & 106 & 50.7 \\
\hline - & Temor a involucrarse en asuntos legales & 79 & 37.8 \\
\hline - & Falta de conocimiento sobre el tema & 72 & 34.4 \\
\hline $\begin{array}{l}\text { Es difícil identificar y tratar los casos en } \\
\text { el desempeño de la práctica médica }\end{array}$ & 45 & 21.5 \\
\hline $\begin{array}{l}\text { Es incómodo identificar y tratar este tipo } \\
\quad \text { de problema }\end{array}$ & 42 & 20.1 \\
\hline
\end{tabular}

- 0 tro (actitud del personal, a los médicos no les agrada hablar del tema, la propia experiencia de violencia, el paciente lo oculta, la justicia no protege a la mujer)

En la práctica médica para referir

\begin{tabular}{llll} 
- & Temor a involucrarse en asuntos legales & 77 & 37.5 \\
\hline - & Desconocimiento sobre lugares de referencia & 73 & 35.6 \\
\hline - & Falta de conocimiento sobre el tema & 71 & 34.6 \\
\hline - & El personal cons privado de la violencia & 70 & 34.1 \\
\hline $\begin{array}{l}\text { en este tipo de problema } \\
\text { - }\end{array}$ & 24 & 11.7 \\
$\begin{array}{l}\text { o tro (falta de apoyo de directores, trámites } \\
\text { mujer tiene miedo, pocos especialistas disponibles }\end{array}$ & \\
& \\
para tratar los casos) & 12 & \\
\end{tabular}

VG : violencia de género

Fuente: $C$ uestionarios del estudio acerca de actitud y conocimiento sobre violencia de género del personal de salud. Ministerio de Salud de N icaragua, 2003 sin embargo, se trata de un proceso no exento de contradicciones.

Resultan congruentes con lo anterior varios datos que revelan que el personal de salud tiene un nivel de conocimiento general sobre la VG: dos tercios de dicho personal tiene una percepción de su prevalencia muy cercana a la realidad nicaragüense, a diferencia de lo que se notifica en el estudio de Sugg y colaboradores, ${ }^{14}$ donde el personal médico, el de enfermería y las auxiliares subestimaron la magnitud del problema en su país. A la vez, un porcentaje aceptable (58.1\%) reconoce el tipo de daños asociados a la VG: físicos, psicológicos y, en menor medida, los sexuales. Interesa destacar el hecho de que el personal que tiene un familiar o amigos cercanos con problemas de VG, presenta una mejor actitud, 3.2 veces más, que el que no tiene esta experiencia, lo que podría estar asociado a una sensibilización a todo lo que implica la problemática de violencia cuando sucede en otros, lo que no necesariamente ocurre cuando uno es el que está involucrado. Por lo tanto, es necesario tener presente que la ausencia o la vivencia de experiencias personales, familiares y culturales pueden fomentar, o bien, según sea el caso, obstaculizar una actitud de rechazo hacia la VG y ello, a su vez, influir sobre la forma en que el personal de salud reacciona ante el paciente. ${ }^{20}$

Otro aspecto que coexiste y, a la vez, entra en contradicción con la actitud de rechazo hacia la VG prevaleciente en este grupo es que alrededor de la tercera parte del personal de salud ha sido víctima o ejecutor de episodios de cualquier tipo de violencia, proporción que se considera alta en comparación con los datos de un estudio realizado en México, que reportó cifras de 13 y $18 \%$ de violencia física/sexual. ${ }^{21}$ Llama más aún la atención que $20 \%$ del personal de salud ejerce violencia hacia su pareja, de tal manera que puede haber contradicciones entre lo que tiene que hacer ante un caso de violencia y lo que hace en realidad, y ello constituye una barrera difícil de eliminar con capacitaciones meramente informativas.

Otro elemento contradictorio con la expresa actitud de rechazo ante la VG se ubicó en la práctica médica como una barrera, tanto para el proceso de identificación como para el de referencia de las víctimas de la VG; es decir, en el hecho de que el personal de salud considerara que la violencia tiene carácter privado $\mathrm{y}$, por ende, de que no identificara el ámbito de los servicios de salud como el lugar donde se tendría que ventilar esta situación. Esta postura se puso de manifiesto no sólo entre el personal de salud, sino también en la institución; en el primer caso, la mitad lo expresó tácitamente, además de que imperaba el temor a involucrarse en asuntos legales (casi la mitad) y a poner en 
riesgo la propia seguridad (37.8\%); en el segundo caso, el personal identificaba ciertas barreras institucionales como el hecho de que la institución no le brindaba el apoyo requerido y la falta de espacios privados para la consulta. Todo lo anterior evidencia un condicionamiento social que rebasa el plano individual y que tiene implicaciones en el abordaje y el tratamiento de los casos de VG. Esto provoca que el personal de salud desconfíe de las víctimas, se sienta incómodo, tenga temor a ofender si hace preguntas a las pacientes o manifieste no poder contribuir a solucionar el problema, como se ha mencionado en otros estudios. ${ }^{16,17,25-27}$

Se podría pensar que una actitud de rechazo ante la VG es el resultado de la existencia de normas y procedimientos para la atención de sus víctimas y de la capacitación que se ha dado al personal en estas áreas. Esto último en parte es cierto, ya que la actitud de rechazo se encontró asociada al personal que mostró un nivel de conocimiento destacado de las Normas de Atención (nivel medio y alto), 4.3 veces más que el personal con conocimiento bajo, lo cual concuerda con los resultados notificados por Méndez y colaboradores. ${ }^{15}$

Asimismo, otro hallazgo del estudio relacionado con el punto anterior es que se encontró asociación de la actitud de rechazo con la profesión médica, pues fue precisamente el personal médico el que mostró tener un mayor nivel de conocimiento de las Normas, encontrándose 6.5 veces más la posibilidad de que este personal tenga una actitud de rechazo hacia la VG en comparación con el de enfermería. Lo anterior difiere con lo registrado por Cann y colaboradores, ${ }^{28}$ quienes encontraron que las mujeres, el personal de enfermería y de salud mental muestran un mayor conocimiento y una actitud más positiva (de rechazo a la violencia) que el personal médico. En el presente estudio no se encontraron diferencias entre sexo y profesión, posiblemente debido a que más de las tres cuartas partes de la plantilla de personal del programa son mujeres y el tamaño de la población de estudio fue pequeño; por este motivo es necesario realizar otras aproximaciones, y una de ellas podría hacerse por medio de métodos cualitativos.

Pero, por otro lado, la capacitación institucional presenta aún limitaciones: solamente un poco más de la tercera parte del personal ha leído las Normas, y éste considera tal hecho como una barrera institucional común para la identificación y la referencia de casos, lo que concuerda con otros trabajos., ${ }^{9,15-17,26,27}$ En el presente estudio más de la mitad de los sujetos refiere que no fue capacitado al ingresar al programa, lo que llama la atención dado que el Informe del Programa Mujer, Salud y Desarrollo de la Organización Paname- ricana de la Salud (OPS) ${ }^{29}$ menciona que se brindó capacitación a personal de salud (1282) y a facilitadoras (90) de nueve SILAIS. Además, solamente $32 \%$ del personal de salud notificó haber recibido capacitación sobre violencia durante la carrera de medicina, porcentaje que se considera bajo, comparado con lo encontrado por Alpert y colaboradores, ${ }^{30,31}$ quienes concluyen que la enseñanza sobre la VG ha aumentado en las escuelas de medicina.

Sin embargo, ha habido una preocupación social por revertir la ausencia de capacitación, lo que explica que $63 \%$ del personal de salud en los SILAIS se haya capacitado posteriormente, dato que se considera alto en comparación con el de otros estudios hechos en México, Canadá y EUA, donde se registraron cifras de personal no capacitado en activo que van desde 72 hasta $90 \%{ }^{15,16,32-34}$

Es evidente la necesidad de contar con normas y procedimientos para la atención de la VG, así como de darlos a conocer y capacitar al personal de salud en este sentido; sin embargo, aun cuando ello suceda y se modifiquen leyes, las actitudes y los comportamientos tienden a seguir siendo regulados por la normativa cultural. No obstante, también es evidente que en Nicaragua esa situación se modificó en alguna medida como resultante del proceso revolucionario que se vivió durante la década de los ochenta. Ello trajo aparejado cambios culturales, sociales y, por ende, institucionales e individuales que han marcado diferencias respecto al resto de los países de América Latina e, inclusive, a EUA. Se ha visto que los protocolos y normas por sí solos no garantizan una adecuada atención. Cann y colaboradores $^{28}$ refirieron deficiencias en el conocimiento sobre la VG en Inglaterra, entre el personal de salud; sin embargo, la actitud de este último fue favorable.

Lo anterior explica que $81.5 \%$ del personal de los SILAIS haya identificado casos, porcentaje que se considera alto en comparación con los de otros estudios, ${ }^{15,16,32-34}$ donde se notifican proporciones de entre 30 y $78 \%$. Por otro lado, se encontró que menos de la mitad del personal de salud realiza preguntas a las pacientes para identificar la VG y que lo hace sólo cuando sospecha de que se trata de un caso, cantidad que se considera baja en relación con los resultados encontrados por Méndez y colaboradores ${ }^{15}(68 \%)$, al igual que por Rodríguez y colaboradores ${ }^{26}$ quienes registraron que $79 \%$ del personal siempre hace preguntas sobre VG a sus pacientes.

A partir de la identificación de las víctimas de VG, el personal de los SILAIS tiene claridad sobre los procedimientos a seguir. Así, las barreras existentes se deben más a problemas organizacionales de la institu- 
ción que a la falta de instancias de referencia adecuadas. El 92.4\% del personal mostró interés en identificar los casos y hacer lo que éstos ameritaban, lo cual puede resultar efectivo si la conducta que éste sigue durante la atención consiste en brindar consejería a las víctimas $(89.5 \%)$ y referirlas a las instituciones correspondientes $(59.9 \%)$, como las Comisarías de la Mujer $(48.8 \%)$ y ONG especialistas en el tema $(38.8 \%)$, o bien con psicoterapeutas especializados (46.8\%). Si se considera que más de la mitad del personal estudiado realiza actividades de consejería y referencia, y ello se relaciona con el número de casos identificados en los últimos tres meses $(64.3 \%)$, se puede concluir que las víctimas recibieron una atención y una orientación adecuadas, las cuales se podrían mejorar. Las áreas de salud mental o el propio programa de violencia del MINSA fueron mencionados por el personal en un bajo porcentaje, lo que puede indicar que este servicio no se oferta en forma óptima. Por otro lado, las Comisarías de la Mujer se encuentran en todos los departamentos de Nicaragua, al igual que las redes de apoyo de las ONG, por lo que esta barrera organizacional puede superarse mediante una mejor coordinación entre las instituciones.

\section{Conclusiones}

Los hallazgos del presente estudio brindan información acerca del conocimiento y la actitud que el personal de salud muestra respecto a la identificación, el manejo y la referencia de las mujeres víctimas de violencia. Esta información es necesaria para el diseño de las intervenciones que se realizan durante el proceso de atención y para mejorar el modelo que se está desarrollando en los centros de salud de atención primaria en Nicaragua.

La VG es un problema de salud pública grave y complejo cuya detección, atención y prevención, así como la transformación de los patrones socioculturales que la han originado y la perpetúan, constituyen una de las prioridades en los planes y programas de protección y fortalecimiento de los derechos humanos y una condición indispensable para avanzar hacia la equidad de género.

En el sector salud, los resultados refuerzan la idea de que la capacitación del personal es clave para una adecuada atención en los servicios de salud, ya que es posible identificar y referir desde cualquier servicio a las mujeres que sufren algún tipo de violencia, siempre y cuando el personal conozca el problema. Para ello es necesario que el estudio sobre la atención a las víctimas de VG se fomente desde las escuelas de medicina y enfermería y se refuerce en las escuelas de salud pública.
Se puede concluir que el personal con un nivel medio-alto de conocimiento sobre las Normas y Procedimientos para la Atención de las Víctimas de VIF tienen una actitud de rechazo ante la VG. Sin embargo, sólo la capacitación no es suficiente: el hecho de que el personal de salud no reconozca en sí mismo un sentimiento y una actitud desfavorable hacia la VG, puede afectar la comunicación médico-paciente. Asimismo, para que en la práctica se observe un cambio significativo es importante que se consolide la capacitación desde la perspectiva de género. La anterior recomendación cobra fuerza si se toma en cuenta que, a pesar de tener un alto porcentaje de actitud de rechazo hacia la VG y de conocimientos sobre las normas de atención, un porcentaje del personal todavía persiste en la idea de que la violencia es un asunto de carácter privado.

Otra de las barreras encontradas es que todavía no se está cumpliendo totalmente con el procedimiento establecido en las normas de atención, las cuales contemplan: identificación, valoración de riesgo, fortalecimiento, orientación, referencia y registro. Para la identificación y la valoración de riesgo se necesita capacitación, privacidad y tiempo en la consulta, mientras que para el fortalecimiento y la orientación es necesario conocer de lugares de referencia. La falta de apoyo por parte de las autoridades de las instituciones y la falta de coordinación institucional limitan la referencia y el seguimiento de las víctimas de VG. El personal prefiere no registrar los casos, lo que hace parecer que éstos aún no se hacen presentes en los servicios de salud; sin embargo, si existe una actitud de rechazo hacia la VG, entonces las barreras que dificultan la atención pueden ser más fáciles de eliminar.

\section{Agradecimientos}

Se agradece la colaboración de todo el personal que aportó sus experiencias y conocimientos para el estudio. A la licenciada Margarita Gurdián, viceministra del MINSA, por facilitar la autorización para la realización del presente estudio, así como a cada una de las personas en los servicios de salud que reunieron al personal y a las que apoyaron en la recolección de los cuestionarios en los SILAIS donde se realizó el estudio. A la licenciada Daysi Fornos, del Centro de Mujeres IXCHEN Estelí, y a la maestra Rosario Valdez, del Instituto Nacional de Salud Pública, por proporcionarnos el cuestionario sobre el estudio del personal médico del IMSS.

Nuestra gratitud al doctor Róger Rodríguez y a la doctora María Lourdes Bolaños, por su valiosa aportación teórica; al señor Filin Rodríguez, por su apoyo en la recolección de los cuestionarios, y a la señora Josefa Jarquín, por el apoyo en la captura de los datos y las correcciones del documento. 


\section{Referencias}

1. Híjar-Medina M, López-López MV, Blanco-Muñoz J. La violencia y sus repercusiones en la salud; reflexiones teóricas y magnitud del problema en México. Salud Publica Mex 1997;39:565-572.

2. García-Moreno C.Violencia contra la mujer : género y equidad en salud.W ashington, DC: 0 rganización Panamericana de la Salud I Harvard Center for Population and Development Studies; 1999. Serie Género y Equidad en la Salud, Publicación 0 casional $N{ }^{\circ} 6$.

3. Fawcett $G$,Venguer T,Vernon R, Pick S. D etección y manejo de mujeres víctimas de violencia doméstica: desarrollo y evaluación de un programa dirigido al personal de salud. México, D.F: Population Council IN O PAL III; 1998; 26:7-30.

4.Valdez-Santiago R, Sanín-Aguirre LH . La violencia doméstica durante el embarazo y su relación con el bajo peso al nacer. Salud Publica Mex 1996:38:352-362

5.Valladares E, Ellsberg M, Peña R, Högberg U, Persson LA. Physical partner abuse during pregnancy:A risk factor for low birth weight in $N$ icaragua. $O$ bstet Gynecol 2002; 100: 700-705.

6. Monemi KA, Peña, R, Ellsberg M, Persson LA.Violence against women increases the risk of infant and child mortality: $A$ case-referent study in $\mathrm{N}$ icaragua. Bull W orld Health 0 rgan 2003;81(1):10-16.

7. De la Fuente R, Medina-Mora ME, C araveo J.Violencia y salud mental: salud mental en México. México, DF: Instituto Mexicano de Psiquiatría / Fondo de Cultura Económica; 1997:232-253.

8. Ellsberg M, Peña R, Herrera A, Lijestrand J,W inkvist A. Confites en el infierno: prevalencia y características de la violencia conyugal hacia las mujeres de N icaragua. Managua:A sociación de Mujeres Profesionales por la Democracia en el Desarrollo; 1996.

9. Ministerio de Salud. N ormas y Procedimientos para la Atención de la Violencia Intrafamiliar. Managua: Dirección de A tención Integral a la Mujer, N iñez y A dolescencia, MIN SA; 2001.

10. Heise L, Pitanguy J, Germain A.Violencia contra la mujer : la carga oculta sobre la salud. W ashington, D C: O rganización Panamericana de la Salud; 1994.

11. O rganización Panamericana de la Salud. Informe mundial de violencia y salud: resumen.W ashington, DC;2002.

12. Morrison AR, 0 rlando MB. El impacto socioeconómico de la violencia doméstica: $C$ hile y $\mathrm{N}$ icaragua. En: Morrison $A R$, Loreto $M$, ed. El costo del silencio: violencia doméstica en las A méricas. W ashington, DC: Banco Interamericano de Desarrollo; 1999:49-99.

13. Ministerio de Salud. Análisis de la situación de salud 1992-1998. Managua: MIN SA/O PS/O MS; 2000.

14. Sugg N K, Thompson RS, Thompson DC, Maiuro R, Rivara FP. Domestic violence and primary care:Attitudes, practices, and beliefs. Arch Fam Med 1999; 8:301-306.

15. Méndez-Hernández P,Valdez-Santiago R,Viniegra-Velázquez L, Rivera-Rivera L, Salmerón-C astro J.Violencia contra la mujer: conocimiento y actitud del personal médico del Instituto Mexicano del Seguro Social, Morelos, México. Salud Publica Mex 2003:45(6):472-482. 16.W aalen J, Goodwin MM, Spitz AM, Petersen R, Saltzman LE. Screening for intimate partner violence by health care providers: Barriers and interventions. Am J Prev Med 2000;19(4):230-237.
17. Gerbert B, Caspers N, Bronstone A, Moe J,A bercrombie P.A qualitative analysis of how physicians with expertise in domestic violence approach the identification of victims. Ann Intern Med 1999;131:578-584

18. Hathaway JE, W illis $G$, Zimmer B. Listening to survivors' voices. Violence A gainst W omen 2002;8(6):687-719.

19. Riquer-Fernández F. Salud y violencia de género: el relato de un intento de acercamiento. En: Gogna M, Ramón S, comp. Experiencias innovadoras en salud reproductiva. La complementación de las ciencias médicas y sociales. Buenos Aires: Centro de Estudios de la Sociedad; 2002:83-103.

20. N ovack DH, Suchman A L, C lark W, Epstein RM, N ajberg E, Kaplan C. Calibrating the physician: Personal awareness and effective patient care. JAMA 1997;278:502-509.

21. Díaz-0 lavarrieta C, Paz F, García de la C adena C, C ampbell J.

Prevalence of intimate partner abuse among nurses and nurses'aides in Mexico. Arch Med Res 2001;32:79-89.

22. Ministerio de Salud.Abordaje Integral de la violencia doméstica desde los Servicios de Salud. Managua: MIN SA; 2002.

23. Ministerio de Salud. Encuesta de Establecimientos de Salud en N icaragua 2000. Managua: MIN SA; 2000.

24. C asales JC .A lgunos aspectos generales de la teoría de las actitudes. En: C asales JC. Psicología So cial. Contribución a su estudio. La Habana, Cuba: Editorial de Ciencias Sociales; 1989:158-209.

25. Sugg N K, Inui T. Primary care physicians' response to domestic violence. 0 pening Pandora's Box. JA MA 1992;233(23):3157-3160. 26. Rodríguez MA, Bauer HA, McLoughlin E, Grumbach K. Screening and intervention for intimate partner abuse. Practices and attitudes of primary care physicians. JAMA 1999;282(5):468-474.

27. Parsons LH, Zaccaro D, W ells B, Stovall TG. Methods of and attitudes toward screening obstetrics and gynecology patients for domestic violence. Am J 0 bstet Gynecol 1995;173:381-387.

28. C ann K, W ithnell S, Shakespeare J, D oll H, Tho mas J. D omestic violence:A comparative survey of level of detection, knowledge, and attitudes in healthcare workers. Public Health 2001;115:89-95.

29. 0 rganización Panamericana de la Salud / 0 rganización Mundial de la Salud. Hacia un modelo integral de prevención y atención de la violencia intrafamiliar: ampliación y consolidación de las intervenciones coordinadas por el Estado y la sociedad civil. Programa Mujer, Salud y Desar rollo. Washington, DC: O PS/O MS; $2001.16 \mathrm{p}$. 30. Alpert EJ, Tonkin A E, Seeherman AM, H oltz HA. Family violence curricula in U.S. medical schools. Am J Prev Med 1998;14:273-282. 31. Alpert EJ, Shannon D, Velonis A, G eorges M, Rich RA. Family violence and public health education.Violence A gainst W omen 2002;8(6):746778.

32. Reid SA, Glasser M. Primary care physicians' recognition of attitudes toward domestic violence. Acad Med 1997;(1):51-53.

33. Ferris LE. Canadian family physicians' and general practitioners' perceptions of their effectiveness in identifying and treating wife abuse. Med Care 1994; 32(12):1163-1172.

34. G roth B, Chelmowski MK, Batson TP. D omestic violence: Level of training, knowledge base and practice among Milwaukee physicians. W MJ 2001;100(1):24-28. 